\title{
Automated design of multi junction solar cells by genetic approach: reaching the $>50 \%$ efficiency target
}

\author{
Slobodan Čičić and Stanko Tomić ${ }^{1}$ \\ Joule Physics Laboratory, School of Computing, Science and Engineering, University of \\ Salford, Manchester M5 4WT, United Kingdom
}

\begin{abstract}
The proper design of the multi-junction solar cell (MJSC) requires the optimisation search through the vast parameter space, with parameters for the proper operation quite often being constrained, like the current matching throughout the cell. Due to high complexity number of MJSC device parameters might be huge, which makes it a demanding task for the most of the optimising strategies based on gradient algorithm. One way to overcome those difficulties is to employ the global optimisation algorithms based on the stochastic search. We present the procedure for the design of MJSC based on the heuristic method, the genetic algorithm, taking into account physical parameters of the solar cell as well as various relevant radiative and non-radiative losses. In the presented model, the number of optimising parameters is $5 M+1$ for a series constrained $M$-junctions solar cell. Diffusion dark current, radiative and Auger recombinations are taken into account with actual ASTM G173-03 Global tilted solar spectra, while the absorption properties of individual SCs were calculated using the multi band $\mathbf{k} \cdot \mathbf{p}$ Hamiltonian. We predicted the efficiencies in case of $M=4$ to be $50.8 \%$ and $55.2 \%$ when all losses are taken into account and with only radiative recombination, respectively.
\end{abstract}

Keywords: Multi Junction Solar Cells, Current Matching, III-V semiconductors, Auger effect, Genetic Algorithm

\footnotetext{
${ }^{1}$ Corresponding author. Tel.: $+44(0) 161-295-3847$.

E-mail address: s.tomic@salford.ac.uk (S. Tomić). 


\section{Introduction}

Among the third generation solar cell concepts, the multijunction solar cells (MJSC) are the only devices with the proven efficiency [1], which exceed the Shockley-Queisser limit [2], in the laboratory and small commercial setups. This is achieved due to much better spectrum matching than in the other $\mathrm{SC}$ concepts $[1,3]$. However, this concept still lacks the detailed theoretical descriptions and understanding of major factors influencing the operation and efficiency of such devices. The majority of MJSC models so far were based on principles of the detailed balance and thermodynamics [4] [5] [6] [7], with recent attempts to address the real SC's material parameters [8]

The main conceptual message of the MJSC and a route to overcome the poor spectral matching of a single-junction SC is to introduce subcells with different energy gaps $\left(E_{g}\right)$ into the device [9]. Such devices consisting of several solar cells (SC), i.e subcells, each of which with different $E_{g}$ are capable of absorbing the photons from different part of the solar spectrum. Generally, this design is achieved by growing semiconductors with different $E_{g}$ on top of each other. The upper subcells are grown with a higher $E_{g}$ and absorb photons with higher energies. Each subcell is also transparent for the photons with energies lower than its $E_{g}$. Such a concept provides for the absorption of the higher energy photons in the upper subcells and prevents them from being absorbed in the subsequent subcells to reduce the thermal losses $[10,11]$. Increasing the number of subcells to the extreme, when the Sun spectrum is divided so that each subcell is illuminated almost with monochromatic light, conceptually will allow the extreme efficiencies $[12,13,14]$. The MJSCs with increased number of junctions, when compered to the single junction $\mathrm{SC}$, have additional significant benefit of lowering the current density, and reduction of $I^{2} R$ series resistance loss. This lowering of currents generated in subcells is due to spectra splitting between subcells.

In order to achieve the highest possible values of solar cell efficiencies, the design parameters have to be optimally selected and tuned. To find the optimal combination of these parameters, we used the drift-diffusion model, with all parameters of III-V semiconductors, like the effective masses, conduction band and valence band density of states, etc., calculated and scaled as a function of the energy gap. This way we have formed a generic material parameter set, which together with the detailed model of radiative and non-radiative (Auger) losses, provides reliable basis for the description of the underlying parameters of MJSC and its subcells. In our analysis we have 
optimised the pn junctions of an MJSC only. We assume the sufficiently good anti-reflective coating with neglected reflection. In order to find the optimal efficiency in such multi-dimensional phase space with conflicting requirements and constrained parameters, we employed the global optimisation strategies using the genetic algorithm as a driver to run the drift diffusion model as a solver.

The main advantages of the genetic algorithm (GA) over other, more conventional optimisation techniques based on gradient methods, is the robustness, stability, and ability to avoid being trapped (quenched) in a local maxima during the search for the global one. The GA does not make any presumptions about the objective function, and can be used safely when the objective function is discontinuous, stochastic, highly nonlinear or has undefined derivatives. Furthermore, the GA is applicable when the parameter space is constrained by highly complex and conflicting requirements or is $N$-dimensional. The GA is very quick in locating the area, in a multidimensional parameter space, around the optimal solution, however unlike gradient based methods, it takes them more time to reach the exact solution within the located area.

The MJSC design parameters which were optimised by the GA are: thicknesses, impurity concentrations, energy gaps and optimal current through the device under the current matching condition. In order to address effect of radiative and non-radiative losses on the MJSC efficiencies, the optimisation was conducted for three characteristic cases. First, with the radiative recombination only taken into account[15] [16]. Next, with the radiative recombination and diffusion current [17]. And finally, with the Auger recombination together with the other two losses[18]. MJSCs were optimised as series constrained, which means optimal currents of individual cells in MJSC are equal. All predictions were carried out with the actual ASTM G173-03 Global tilted solar spectrum [19] while the absorption spectra for each subcell were calculated using the kppw parallel code [20]. Our results reveal the influence of each type of losses to the overall efficiency. The model based on the genetic algorithm predicts the efficiency of $55.2 \%$ and above in the case 4-junction $\mathrm{SC}$ in the radiative limit. This efficiency drops to $50.77 \%$ when all losses are taken into account in the case of series constrained MJSC. 


\section{Methodology}

\subsection{Theory}

In the general form, the electron-hole pair generation in $m^{\text {th }}$ solar cell of an MJSC can be written as:

$$
\begin{aligned}
g^{(m)}(\lambda, z) & =\Phi(\lambda)[1-R(\lambda)]\left(\prod_{k=1}^{m}\left[1-R_{\mathrm{k}}(\lambda)\right]\right) \\
& \times\left(\prod_{k=1}^{m-1} e^{-\alpha_{\mathrm{k}}(\lambda)\left(z_{\mathrm{k}+1}-z_{\mathrm{k}}\right)}\right) \\
& \times \alpha_{\mathrm{m}}(\lambda) e^{-\alpha_{\mathrm{m}}(\lambda)\left(z-z_{\mathrm{m}}\right)}
\end{aligned}
$$

where $\Phi(\lambda)$ is the solar photon flux, $R(\lambda)$ is the reflection coefficient on surface of the MJSC, $R_{\mathrm{k}}(\lambda)$ is the reflection between two, $k^{\text {th }}$ and $k+1^{\text {st }}$, subcell in MJSC, $\alpha_{\mathrm{k}}(\lambda)$ is the absorption of $k^{\text {th }}$ subcell. In our procedure, we assumed $R(\lambda)=0$ and all $R_{\mathrm{k}}(\lambda)=0$, therefore the generations in the first, second, ..., and $m^{\text {th }}$ subcell, can be rewritten as:

$$
\begin{gathered}
g^{(1)}(\lambda, z)=\Phi(\lambda) \alpha_{1}(\lambda) e^{-\alpha_{1}(\lambda)\left(z-z_{1}\right)} \\
g^{(2)}(\lambda, z)=\Phi(\lambda) e^{-\alpha_{1}(\lambda)\left(z_{2}-z_{1}\right)} \\
\times \alpha_{2}(\lambda) e^{-\alpha_{2}(\lambda)\left(z-z_{2}\right)} \\
g^{(m)}(\lambda, z)= \\
\quad \\
\times \alpha_{m}(\lambda)\left(\prod_{k=1}^{m-1} e^{-\alpha_{m}(\lambda)\left(z-z_{m}\right)} .\right.
\end{gathered}
$$

However, if the $m^{\text {th }}$ subcell is not thick enough, not all the photons are going to be absorbed and some of the higher energy photons will pass to the $m+1^{\text {st }}$ subcell, Fig. 1. In such a situation, in addition to transmitted photons, the subsequent subcell absorbs photons with energies that are higher than its band gap and lower than upper subcell's $E_{\mathrm{g}}$, and properly attenuated for the thickness of the preceding regions in the MJSC stack too, see Fig. 1. The 
absorptions coefficients $\alpha(\lambda)$ are calculated using the parallel kppw code [20]. Actual III-V material parameters, such as refractive index, relative dielectric constant, effective masses and effective density of states were calculated from the $\mathbf{k} \cdot \mathbf{p}$ theory and their functional dependence on $E_{g}$ is given in the Appendix.

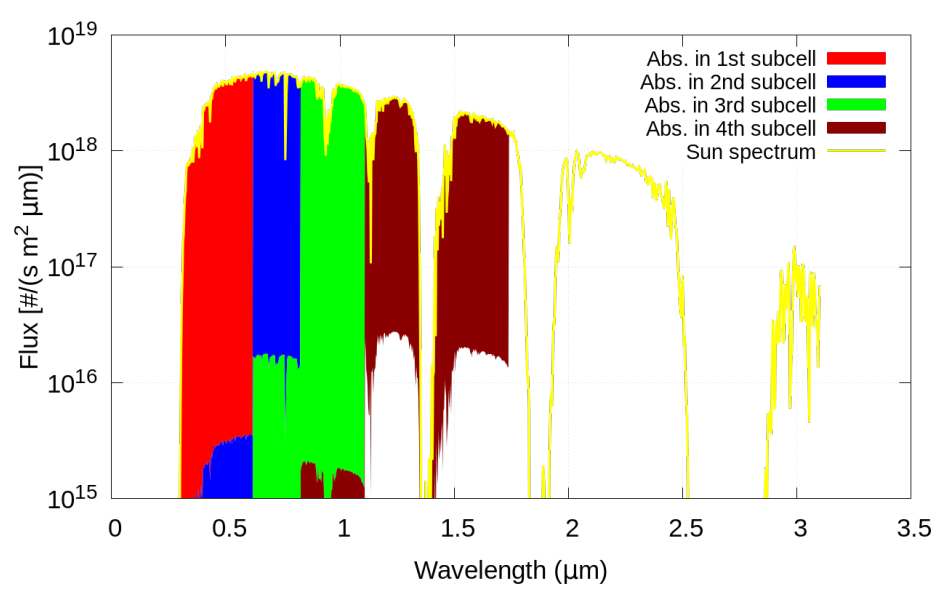

Figure 1: ASTM G173-03 Global tilted solar spectra and attenuated portions of this spectra in each of 4 subcells in 4-junction MJSC. It corresponds to the 4-junctions MJCS analysed in Fig 3(c)

The general expression for the current generated in $m^{\text {th }}$ subcell is then:

$$
J_{\nu}^{(m)}=q \int_{z_{m, l}}^{z_{m, u}} \int_{\lambda_{l}}^{\lambda_{u}} g^{(m)}\left(\lambda^{\prime}, z^{\prime}\right) d \lambda^{\prime} d z^{\prime} .
$$

where $z_{m, l}$ and $z_{m, u}$ are the coordinates of the lower and upper edge of the $m^{\text {th }}$ subcell and $\lambda_{l}$ and $\lambda_{u}$ are the lower and upper light wavelength absorbed in the $m^{\text {th }}$ subcell; for ASTM G173-03 $\lambda_{l}=280 \mathrm{~nm}$ and $\lambda_{u}=4000 \mathrm{~nm}$ [19]. In Eq. 5 index $\nu \in\{n, \mathrm{dr}, p\}$, where $n$, $\mathrm{dr}$ and $p$ represent minority carrier current in quasi-neutral $p$ - region, majority electron and hole current in depletion region and minority carrier current in quasi-neutral $n-$ region, respectively. The expressions for currents in different regions of MJSC depend on their geometry, material type, and impurities. For the $p n$ homojunction, those are the textbook expressions, and together with the boundary conditions, can be found elsewhere [17, 21, 22, 23].

Now the short circuit current in $m^{\text {th }}$ subcell, $J_{\mathrm{SC}}^{(m)}$, can be calculated as: 


$$
J_{\mathrm{sc}}^{(m)}=J_{n}^{(m)}+J_{\mathrm{dr}}^{(m)}+J_{p}^{(m)}
$$

where $J_{n}$ is the electron minority current in the quasi-neutral $p$ region, $J_{\mathrm{dr}}$ is the electron and hole majority current in the depletion region and $J_{p}$ is the hole minority current in the quasi-neutral $n$ region.

Various losses existing in the MJSC are accounted in the form of the diode equation:

$$
\begin{gathered}
J_{0}^{(m)}=J_{\text {sat }}^{(m)}\left(e^{\frac{q V}{k_{\mathrm{B}} T}}-1\right) \\
J_{\text {sat }}^{(m)}=J_{0, \text { Rad. }}^{(m)}+J_{0, \text { Dark }}^{(m)}+J_{0, \text { Aug. }}^{(m)}
\end{gathered}
$$

where $J_{0, \text { Rad. }}[15][16], J_{0, \text { Dark }}[17]$ and $J_{0, \text { Aug. }}[18]$ are the diffusion dark current, the radiative recombination current and the Auger recombination current, respectively, given as:

$$
J_{0, \operatorname{Rad}}^{(m)}=q\left[w_{n}^{(m)}+w_{p}^{(m)}\right] \frac{8 \pi n_{i}^{(m) 2}}{h^{3} c^{2}}\left[\int_{0}^{\infty} \frac{\alpha^{(m)}(h \nu)^{2}}{e^{\frac{h \nu}{k_{\mathrm{B}}}}} d(h \nu)\right]
$$

where, $\alpha^{(m)}(h \nu)$ is the absorption coefficient of relevant materials, $n_{i}^{(m)}$ is the intrinsics carrier concentration, and $w_{n}^{(m)}+w_{p}^{(m)}$ is the thickness of $m^{\text {th }}$ subcell depletion region;

$$
\begin{aligned}
J_{0, \text { Dark }}^{(m)} & =\frac{q D_{n} n_{0}}{L_{n}}\left[\frac{\frac{S_{n} L_{n}}{D_{n}} \cosh \frac{z_{p}^{(m)}-w_{p}^{(m)}}{L_{n}}+\sinh \frac{z_{p}^{(m)}-w_{p}^{(m)}}{L_{n}}}{\frac{S_{n} L_{n}}{D_{n}} \sinh \frac{z_{p}^{(m)}-w_{p}^{(m)}}{L_{n}}+\cosh \frac{z_{p}^{(m)}-w_{p}^{(m)}}{L_{n}}}\right] \\
& +\frac{q D_{p} p_{0}}{L_{p}}\left[\frac{\frac{S_{p} L_{p}}{D_{p}} \cosh \frac{z_{n}^{(m)}-w_{n}^{(m)}}{L_{p}}+\sinh \frac{z_{n}^{(m)}-w_{n}^{(m)}}{L_{p}}}{\frac{S_{p} L_{p}}{D_{p}} \sinh \frac{z_{n}^{(m)}-w_{n}^{(m)}}{L_{p}}+\cosh \frac{z_{n}^{(m)}-w_{n}^{(m)}}{L_{p}}}\right]
\end{aligned}
$$

where $n(p)_{0}$ is the electron (hole) concentration in equilibrium, $S_{n(p)}$ is the surface recombination velocity, $L_{n(p)}$ is the diffusion length for minority carriers, $D_{n(p)}$ is the diffusion constant given by Einsteins relationship, and $z_{n(p)}^{(m)}$ is the thickness of $n(p)$ layer and $z_{n(p)}^{(m)}-w_{n(p)}^{(m)}$ is the thickness of $n(p)-$ quasineural region in the $m^{\text {th }}$ subcell. All parameters in Eq. 10, $S_{n(p)}, D_{n(p)}, L_{n(p)}, n(p)_{0}$, are the $m^{\text {th }}$ subcell's material dependent. The total current in a solar cell has 
two contribution: the current due to solar irradiation and the dark current, $J_{0 \text {,Dark }}^{(m)}$, due to voltage across the SC. Voltage drives majority carriers in the opposite direction comparing to minority carriers driven by light. Therefore, it is accounted as a loss.

In the Auger current we took into account the two most prominent processes in III-V semiconductors: (i) CHCC Auger recombination process in which a conduction electron recombines with a valence hole, with the released energy exciting a second conduction electron to a vacant higher conduction state and (ii) CHHS Auger recombination process in which a conduction electron recombines with a valence hole with the released energy exciting a valence hole to the spin-split-off band; in both cases such that energy and momentum are conserved:

$$
J_{0, \text { Aug. }}=q\left[C_{\mathrm{CHCC}} N_{d}\left(z_{n}^{(m)}-w_{n}^{(m)}\right)+C_{\mathrm{CHHS}} N_{a}\left(z_{p}^{(m)}-w_{p}^{(m)}\right)\right] n_{i}^{2}
$$

where $C_{\mathrm{CHCC}}$ and $C_{\mathrm{CHHS}}$ are the Auger coefficients given as:

$$
C_{\mathrm{CHCC}}=a\left(\frac{m_{e}^{*}}{m_{\mathrm{hh}}^{*}}\right) \frac{\hbar^{3}}{m_{e}^{* 2} \sqrt{E_{g}}} \frac{\left\langle\epsilon_{c}\right\rangle}{\left(k_{\mathrm{B}} T\right)^{3 / 2}}\left(\frac{e^{2}}{\varepsilon_{0} \varepsilon_{\mathrm{r}} E_{g}}\right)^{2} e^{-\epsilon_{\mathrm{th}} / k_{\mathrm{B}} T}
$$

and

$$
\epsilon_{\mathrm{th}}=\left(\frac{m_{e}^{*}}{m_{\mathrm{hh}}^{*}}\right) E_{g} \frac{\left(\Delta+2 E_{g}\right)\left(2 \Delta+3 E_{g}\right)}{\left(\Delta+3 E_{g}\right)\left(\Delta+E_{g}\right)}
$$

where $m_{e}^{*}$ and $m_{\mathrm{hh}}^{*}$ are the electron and heavy hole effective mass respectively, $E_{g}$ is the energy gap, $\Delta$ is the spin-orbit splitting energy, $\varepsilon_{0}$ is the vacuum permitivity, $\varepsilon_{\mathrm{r}}$ is the relative permitivity. If $E_{g}>\Delta$ then $a=(2 \pi)^{5 / 2}$, and $\left\langle\epsilon_{c}\right\rangle=\frac{3}{2} k_{\mathrm{B}} T$, and

$$
\begin{gathered}
C_{\mathrm{CHHS}}=\frac{216 \pi^{5 / 2} \hbar^{3} e^{4} m_{e}^{*}\left(\Delta+E_{g}\right)^{2} \sqrt{k_{\mathrm{B}} T}}{\varepsilon_{0}^{2} \varepsilon_{\mathrm{r}}^{2} m_{\mathrm{hh}}^{*} m_{\mathrm{so}}^{*} E_{g}^{2} \sqrt{\epsilon_{\mathrm{th}}}} e^{-\epsilon_{\mathrm{th}} / k_{\mathrm{B}} T} \\
\epsilon_{\mathrm{th}}=\left(\frac{m_{\mathrm{so}}^{*}}{m_{\mathrm{hh}}^{*}}\right) E_{g} \frac{\left(E_{g}-\Delta\right)}{\left(E_{g}+\Delta\right)\left(3 E_{g}-2 \Delta\right)}
\end{gathered}
$$

where $m_{\mathrm{so}}^{*}$ is the effective mass of the spin-orbit band.

In $n$-type material electrons are the majority carriers. Hence, $\mathrm{CHCC}$ is the most common type of Auger recombination. On the other hand, in $p$ - type material the majority carriers are holes, and CHHL and CHHS would be the most dominant recombination processes. Since in typical III-V 
semiconductors, with energy gap larger then spin-orbit splitting, CHHS is more dominant type of Auger recombination, and CHHL can be neglected.

The optimal current is then:

$$
J_{\mathrm{opt}}^{(m)}=J_{\mathrm{sc}}^{(m)}-J_{\mathrm{sat}}^{(m)}\left(e^{\frac{q V_{\mathrm{opt}}^{(m)}}{k_{B} T}}-1\right) .
$$

In our procedure we optimised the series constrained MJSC where all the subcells have equal currents, while voltages are different and independent from each other. Therefore, it is computationally more efficient to express voltage as a function of current and maximise the optimal current. The above equation, Eq. 16 now becomes:

$$
V_{\mathrm{opt}}^{(m)}=\frac{k_{\mathrm{B}} T}{q} \ln \left(\frac{J_{\mathrm{sc}}^{(m)}-J_{\mathrm{opt}}^{(m)}}{J_{\mathrm{sat}}^{(m)}}+1\right) .
$$

Under the current matching condition, currents in each subcell are the same, i.e. $J_{\mathrm{opt}}^{(1)}=J_{\mathrm{opt}}^{(2)}=\ldots=J_{\mathrm{opt}}^{(M)}=J_{\mathrm{opt}}$ giving the expression for the optimal voltage as a sum of optimal voltages in the individual SCs of a MJSC:

$$
V_{\mathrm{opt}}=\frac{k_{\mathrm{B}} T}{q} \sum_{i=1}^{M} \ln \left(\frac{J_{\mathrm{sc}}^{(i)}-J_{\mathrm{opt}}}{J_{\mathrm{sat}}^{(i)}}+1\right) .
$$

The optimal output power of a MJSC is then:

$$
P_{\mathrm{opt}}=V_{\mathrm{opt}} J_{\mathrm{opt}}
$$

and the efficiency of the device is

$$
\eta=P_{\mathrm{opt}} / P_{\mathrm{Sun}}
$$

where $P_{\text {Sun }}=100 \mathrm{~mW} \mathrm{~cm}^{-2}$, is the power of the Sun photons at the Earth surface, with the AM1.5 and direct irradiance. All III-V material parameters entering currents in the Eq. 18 were calculated from $\mathbf{k} \cdot \mathbf{p}$ theory and expressed as a function of energy gap $\left(E_{g}\right)$. The accuracy is tested by comparison to the material parameter sets collected in[24, 25]. 


\subsection{Global optimisation of MJSC: genetic algorithm approach}

The optimisation of a MJSC is being done through the stochastic variation and selection of its parameters in order to achieve the maximal efficiency of the MJSC, Eq. 20, as a target function. Due to the complexity of MJSCs, a large number of optimising parameters creates a huge parameter space. Such parameter space is often uneven and could contain plenty of local maxima/minima that could cause a quenching of the search algorithm, which must be prevented in the successful optimisation [26]. It makes it more difficult for most of the search methods, particularly those based on the gradient methods, to locate the global maximum. However the search algorithms based on stochastic methods could reliably avoid such traps. Because of that we opt here to use the genetic algorithm (GA) in our optimisations of MJSCs $[27,28]$.

Parameters that we have optimised in our procedure using the GA are: $J_{\text {opt }}$, the optimal current density (one parameter), $z_{\mathrm{p}}, z_{\mathrm{n}}$ the thicknesses of emitter and base in all subcells ( $2 M$ parameters), the emitter, $N_{\mathrm{a}}$, and base, $N_{\mathrm{d}}$, doping concentration of all subcells ( $2 M$ parameters), and the material energy gap $E_{\mathrm{g}}$ of all subcells ( $M$ parameters). In case of series constrained MJSC the number of optimising parameters is $5 M+1$, where $M$ is the number of subcells in the MJSC.

Since the population of $N_{I}$ individuals in the $i^{\text {th }}$ generation of the GA is given by a vector, $\mathbf{X}^{(i)}=\left\langle x_{1}^{(i)}, x_{2}^{(i)}, \ldots, x_{N_{I}}^{(i)}\right\rangle$, where $x_{k}^{(i)}$ is the $k^{\text {th }}$ individual in $i^{\text {th }}$ generation; we can easily represent the $5 M+1$ MJSCs parameters as a single, $k^{\text {th }}$, individual of population in the $i^{\text {th }}$ generation as vector: $x_{k}^{(i)}=\left\langle J_{\mathrm{opt} ; 1}, z_{\mathrm{p} ; 1 . . M}, w_{\mathrm{n} ; 1 . . M}, N_{\mathrm{a}, 1 . . M}, N_{\mathrm{d}, 1 . . M}, E_{\mathrm{g} ; 1 . . M}\right\rangle_{k}^{(i)}$. The efficiency of MJSC, Eq. 20, in $i^{\text {th }}$ generation is then rewritten as $\eta^{(i)}=\max _{k}\left\{f\left[x_{k}^{(i)}\right]\right\}$. The nature of the GA, as explained below, provides for, $\eta^{(i+1)} \geq \eta^{(i)}$. The overall maximum in the procedure is then reached for the large number of generations, $N_{G}$, i.e., $\eta_{\text {opt }}=\lim _{i \rightarrow N_{G}}\left\{\max _{i} f\left[\mathbf{X}^{(i)}\right]\right\}$. In our procedure we always set $N_{G}$ to provide for the convergency of $\eta_{\text {opt }}$ better than $10^{-5}$.

Generally, each GA algorithm consist of three operators that prevent quenching into local minima: selection, crossingover and mutation.

The selection operator chooses individuals for reproduction (crossingover and mutation) based on their fitness, i.e., in MJSC based on the efficiency. Before taking part in the reproduction, individuals have to be encoded. Each actual material parameter is being converted into a binary string, genome, while a set of encoded parameters is called the chromosome. The number of 
bits used to encode parameters of MJSC can vary and in our case it is set to 5 . It means that each genome has a length of $2^{5}$ and each chromosome $(5 \mathrm{M}+$ 1) $\cdot 2^{5}$ in the case of series constrained MJSC. The chromosome selections is based on the roulette wheel algorithm, where each member of the population has a roulette wheel slot with a size proportional to its fitness. The relative fitness of the $k^{\text {th }}$ individual in any given generation is $f\left(x_{k}\right) / \bar{f}$, where $\bar{f}=$ $\sum_{k=1}^{N_{I}} f\left(x_{k}\right) / N_{I}$ is the average fitness. In our case the function, $f$, is the efficiency function, $\eta$, Eq. 20. This way chromosomes which provide better fitness will take part in reproduction more often and, therefore, have more offsprings.

Crossingover provides for two parent individuals to be combined by exchanging parts of their chromosomes in order to create two new offsprings. This way the new offspring can have better fitness than their parents, i.e., provides for $\eta^{(i+1)} \geq \eta^{(i)}$. Since crossingover is the main search driver throughout the parameter space in the GAs, its probability must be larger than 0.5. In our case it is set to 0.85 to provide for rapid convergence.

Mutation changes chromosomes randomly and, thus, helps avoiding GA being trapped in the local maximum before reaching the global one. Its main purpose is to maintain a certain level of the genetic diversity of population to prevent premature convergence. Since crossingover combines already existing chromosomes, it could converge to a local maximum. Therefore, mutation operator is essential in search for the global maximum. In our case mutation probability is set to 0.005 , although it can be set to variable mutation rates between 0.0005 and 0.25 .

\section{Results and Discussions}

In order to achieve the highest possible efficiency, the SC's short-circuit current has to be maximized and the SC's losses minimized, while the light should be absorbed as much as possible. Expressions that correctly describe these contradicting requirements are very complex and it is very difficult to identify how SC's parameters are correlated, if at all, between themselves when maximising the efficiency. For example, in order to have higher open circuit voltages in solar cells, higher doping is required, which at the same time can elevate some losses, i.e., the Auger current [18]. On the other hand to suppress losses due to the diffusion dark current we need to maximize the impurity concentrations [17]. Materials with higher energy gaps allow higher open circuit voltages and lower losses, whereas, at the same time, reduce 
short circuit currents. Moreover, in 2- junction SC there are 11 material parameters, while for example in the case of 4 -junction $\mathrm{SC}$ the number of parameters for optimisation is increasing quickly to 21. Therefore, heuristic optimisation is crucial for solving problems as complex as this one. Essentially, the GA drives the evaluation of the fitness-function, $\eta_{\mathrm{opt}}$, for each set of SC's physical parameters and then applies the genetic operators to create the new set of parameters inside the population with better fitnesses.

The total thickness of each MJSC analysed in this section was kept at 5 $\mu \mathrm{m}$. This value was chosen to be long enough to provide for the absorption of almost all Sun's photons and in the same time few times shorter than diffusion length, which would negatively affect the carrier collection otherwise. The minority carrier diffusion lengths are taken as $L_{\mathrm{n}}=3 \mu \mathrm{m}$ and $L_{\mathrm{p}}=10 \mu \mathrm{m}$, and surface recombination velocity $S_{n}=S_{p}=1000 \mathrm{~cm} / \mathrm{s}$. In the Einstein's relationships for the diffusion constants of $n(p)$ material we assume the mobility of minority carriers as a function of acceptor(donor) doping concentrations as: $\log \mu_{n}=0.16\left(\log N_{\mathrm{a}}\right)^{2}-5.93 \log N_{\mathrm{a}}+58$ and $\log \mu_{p}=-0.0575 \log N_{\mathrm{d}}+3.416$ [29]. All predictions presented are obtained at $T=300 \mathrm{~K}$.

In Figure 2 we show the maximum efficiency of MJSCs as a function of the number of junctions, $M$. We have examined internal losses of MJSC at various levels of sophistication and their effect on the efficiency in case of: (i) radiative loses, (ii) radiative and diffusion losses, and (iii) radiative, diffusion and Auger losses. For each of these regimes and for each number of junctions, $M$, as one of the outputs of our optimisation procedure we plot in the same graph the optimal arrangements of $E_{g}$ 's (symbols) for each subcell in the MJSCs. It is evident that the effect of the diffusion dark current is very small on the efficiency for all MJSCs considered. The effect of the Auger recombination reduces the maximal efficiency in all MJSCs considered for about $\approx 10 \%$ relative to the radiative limit. Such a large reduction in the efficiency shouldn't be neglected in the design of the MJSCs, and it can only be more pronounced at the elevated temperatures (e.g. $T>300 \mathrm{~K}$ ), often encountered in real operating conditions. Moreover, it is important to note that when the Auger recombination is taken into account, optimal energy gaps for each subcell are slightly higher, and different from those predicted in the radiative limit. This is the consequence of $J_{0, \mathrm{~A}}^{(m)} \propto e^{-E_{g}^{2} / k_{\mathrm{B}} T}$, i.e., the large $E_{g}$ tends to reduce the contribution of the Auger losses to the overall losses in the device, hence maximising the efficiency.

In Table 1 we have summarised our predictions of the maximal efficiency 


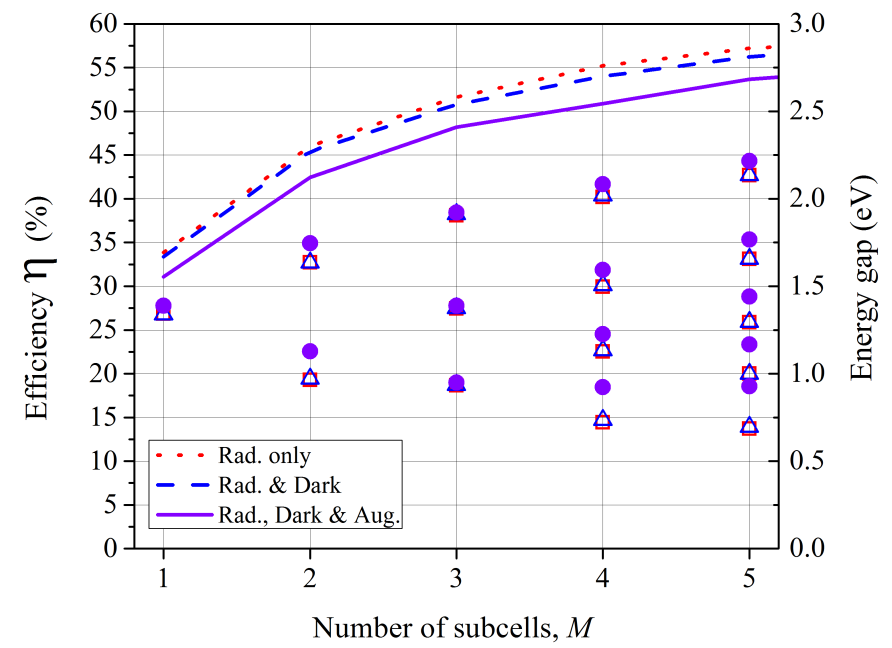

Figure 2: Maximum efficiency of MJSC as a function of the number of junctions (left axes) and the optimal arrangement of the $E_{g}$ (right axes): (i) for radiative limit (dotted line for $\eta$ and open squares for $E_{g}$ ), (ii) for diffusion dark and radiative losses (dashed line for $\eta$ and open triangles for $E_{g}$ ), and (iii) for the radiative, diffusion dark and Auger losses (solid line for $\eta$ and solid circles for $E_{g}$ ). 

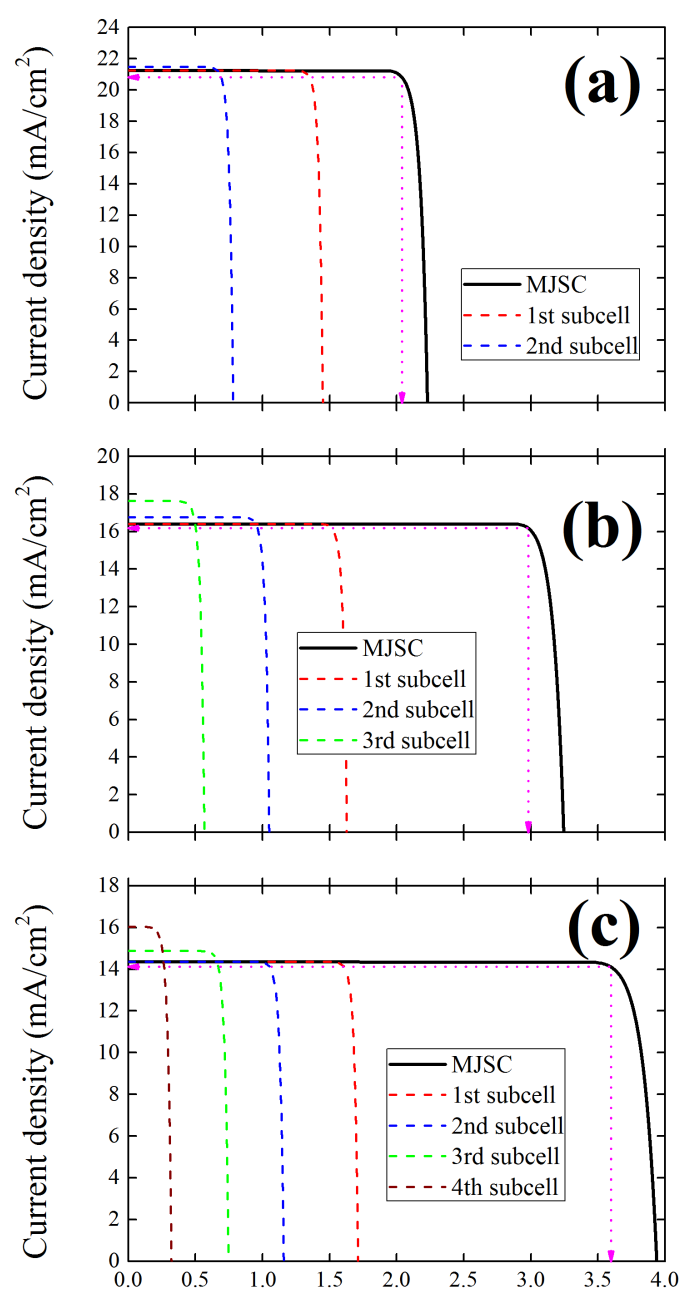

Voltage $\mathrm{V}(\mathrm{V})$

Figure 3: $J V$ curve for: (a) 2-junction solar cell with $J_{\text {opt }}=20.81 \mathrm{~mA} / \mathrm{cm}^{2}$ and $V_{\text {opt }}=2.04$ $\mathrm{V}$, (b) 3 -junction solar cell with $J_{\mathrm{opt}}=16.19 \mathrm{~mA} / \mathrm{cm}^{2}$ and $V_{\mathrm{opt}}=2.98 \mathrm{~V}$ and (c) 4 -junction solar cell with $J_{\mathrm{opt}}=14.11 \mathrm{~mA} / \mathrm{cm}^{2}$ and $V_{\mathrm{opt}}=3.60 \mathrm{~V}$. 
Table 1: Comparison of 2-junction, 3-junction and 4-junction SCs with experimental results [30]

\begin{tabular}{c|cccc}
\hline$\eta[\%]$ & Rad. only & Rad. \& Dark & Rad., Dark \& Aug. & Exp. III-V \\
\hline $2 J S C$ & 46.0 & 45.4 & 42.5 & $31.6 \pm 1.5[31]$ \\
$3 J S C$ & 51.6 & 50.8 & 48.3 & $37.9 \pm 1.2[32]$ \\
$4 J S C$ & 55.2 & 54.0 & 50.8 & $/$ \\
\hline
\end{tabular}

for 2-, 3-, and 4- junction MJSCs, when different loss mechanisms are taken into account during the optimisation, as well as available experimental data of the best MJSC's [30], with the equivalent number of junctions and at one Sun light concentration. Significant difference can be observed between the maximally predicted and efficiencies of actual solar cells devices, Tab. 1, highlighting the deviation of the spectrum matching in actual devices from ideal one. Our optimisation procedure can predict exactly the optimal energy gaps and also the optimal thickness of each subcell in order to achieve almost identical short circuit currents through the device, Fig. 3. For example we have achieved very small differences in our short circuit currents between subcells; in 2- junctions MJSC $J_{\mathrm{sc} 1}=21.253 \mathrm{~mA} \mathrm{~cm}{ }^{-2}$ v. $J_{\mathrm{sc} 2}=21.484$ $\mathrm{mA} \mathrm{cm}{ }^{-2}$, Fig. 3 (a); in 3- junctions MJSC $J_{\mathrm{sc} 1}=16.407 \mathrm{~mA} \mathrm{~cm}^{-2}$ v. $J_{\mathrm{sc} 2}=16.761 \mathrm{~mA} \mathrm{~cm}^{-2}$ v. $J_{\mathrm{sc} 3}=17.625 \mathrm{~mA} \mathrm{~cm}^{-2}$, Fig. 3 (b); and in 4- junctions MJSC $J_{\mathrm{sc} 1}=14.362 \mathrm{~mA} \mathrm{~cm}{ }^{-2} \mathrm{v} . J_{\mathrm{sc} 2}=14.357 \mathrm{~mA} \mathrm{~cm}{ }^{-2} \mathrm{v}$. $J_{\mathrm{sc} 3}=14.883 \mathrm{~mA} \mathrm{~cm}^{-2}$ v. $J_{\mathrm{sc} 4}=16.046 \mathrm{~mA} \mathrm{~cm}^{-2}$, Fig. 3 (c). Such small deviations between $J_{\mathrm{sc}}$ 's and between $J_{\mathrm{sc}}$ 's and $J_{\mathrm{opt}}$ suggests efficient use of photo created carriers and its maximised contribution towards the current. That proves the efficiency of the optimisation algorithm, too.

On the other hand, in actual devices, subcells in a MJSC have significantly different short circuit currents, either due to energy gap availability from certain material combinations, or due to approximated thicknesses of subcells. For the record efficiency devices [30], the measured short circuit currents are: $J_{\mathrm{sc}}=14.30 \mathrm{~mA} \mathrm{~cm} \mathrm{~cm}^{-2}$ for 2- junctions MJSC reported in [31] and $J_{\mathrm{sc}}=15.25$ $\mathrm{mA} \mathrm{cm}{ }^{-2}$ in 3- junctions MJSC reported in [32]. The overall short circuit current in series constrained MJSC is limited by the lowest $J_{\mathrm{sc}_{i}}$ of individual subcells. The reason for the low overall short circuit current could be in one under-performing subcell and below optimal spectral matching. In Alta Devices ${ }^{\circledR}$ 2- junction MJSC the $V_{\text {oc }}=2.547 \mathrm{~V}$, [31] is higher in comparisons to our predicted, $V_{\text {oc }}=2.229 \mathrm{~V}$. This is due to photon recycling [33, 34], which allows the higher voltages, and is not currently taken into account in 
our model. However, in their device based on InGaP/GaAs tandem the short circuit current is low due to relatively high $E_{g}$ of the constituent materials. Relatively high $E_{g}$ is another reason for the above mentioned high $V_{\text {oc }}$ in the actual device. In Sharp ${ }^{\circledR} 3$ - junction MJSC the $V_{\text {oc }}=3.014 \mathrm{~V}$ [32] is lower in comparison to our predicted $V_{\text {oc }}=3.245 \mathrm{~V}$. It is not surprising that with the increase of the number of junctions the availability of materials and tuning between relevant parameters in actual devices become more demanding. It suggests also that our algorithm successfully suppressed major losses during the optimisation procedure, and opens room for further improvements in MJSC designs.

In order to asses the validity of our method, we performed the optimisation procedures with parameters of the actual devices [31, 32]. In Alta Device ${ }^{\circledR}$ 2-junction SC [31] the upper subcell is made of $\operatorname{In}_{0.49} \mathrm{Ga}_{0.51} \mathrm{P}$, lattice matched at GaAs, with $E_{g}=1.9 \mathrm{eV}$ [24] and the lower subcell is GaAs, with $E_{g}=1.42 \mathrm{eV}$. Taking the value of energy gaps as the only data available, we have fixed them in our model and we let the rest of MJSC's parameters to be optimised. The predicted efficiency was $32.34 \%$, which almost coincide with the measured, $31.6 \pm 1.5$ [31]. If we compare values of $V_{\text {oc }}$ between the real device and the one from our model, i.e., $V_{\mathrm{oc}}=2.547 \mathrm{~V}$, [31] versus $V_{\text {oc }}=2.355 \mathrm{~V}$; such small difference can be attributed to the photon recycling effect existing in the real devices and omitted in our model. The Sharp ${ }^{\circledR}$ 3-junction SC [32], has the configuration of two upper subcells the same as in Alta Device ${ }^{\circledR}$ followed with $\operatorname{In}_{0.53} \mathrm{Ga}_{0.47} \mathrm{As}$ as the lowest subcell with $E_{g}=0.74 \mathrm{eV}$ [24]. The efficiency predicted by our optimisation is $38.1 \%$, which is again very close to measured, $37.7 \%$ [32]. The $J V$ characteristics are presented in Fig. 4. Comparing results on MJSCs optimised with fixed $E_{g}$ 's, dictated by actual material combinations in existing devices, Fig. 4, with those fully unconstrained, Fig. 3 (a-b), the advantage of heuristic optimisation is obvious. When all parameters are unconstrained the optimal efficiency is dramatically increased, for $\sim 10 \%$, due to much better adjustment of subcells absorption to the solar spectrum. If all subcells in the MJSC are properly optimised, the deviations between their short-circuit currents, $J_{\mathrm{sc}}^{(m)}$, should be very small. Since in series constrained solar cells the lowest subcell's current defines the output current, a significant difference in short circuit currents leads to non-optimal carrier extraction. Those carries that are not extracted recombine and elevate losses. By detailed inspection of the attenuated portions of sun spectra absorbed in each subcell, Fig. 5, it can be observed how absorbing more photons does not necessarily lead to the higher 
Table 2: Parameters of series constrained 2-junction, 3-junction and 4-junction SCs with diffusion dark current, radiative and Auger recombination taken into account

\begin{tabular}{||c||c|c|c|cc|cc||c||}
\hline \hline$M$ & $m$ & $E_{g}(\mathrm{eV})$ & $z_{\mathrm{sc}}(\mu m)$ & $z_{p}(\mu m)$ & $z_{n}(\mu m)$ & $N_{a}\left(\mathrm{~cm}^{-3}\right)$ & $N_{d}\left(\mathrm{~cm}^{-3}\right)$ & $\eta(\%)$ \\
\hline 2 & 1 & 1.740 & 3.703 & 0.018 & 3.685 & $5.046 \times 10^{18}$ & $6.819 \times 10^{18}$ & 42.462 \\
& 2 & 1.136 & 1.297 & 0.021 & 1.276 & $6.881 \times 10^{17}$ & $1.089 \times 10^{17}$ & \\
\hline 3 & 1 & 1.922 & 1.727 & 0.023 & 1.703 & $6.138 \times 10^{18}$ & $7.884 \times 10^{18}$ & 48.276 \\
& 2 & 1.387 & 1.993 & 0.032 & 1.961 & $7.667 \times 10^{17}$ & $6.540 \times 10^{17}$ & \\
& 3 & 0.949 & 1.280 & 0.044 & 1.236 & $1.679 \times 10^{17}$ & $6.340 \times 10^{16}$ & \\
\hline 4 & 1 & 2.010 & 1.427 & 0.016 & 1.411 & $9.356 \times 10^{18}$ & $5.951 \times 10^{18}$ & 50.777 \\
& 2 & 1.502 & 1.119 & 0.097 & 1.022 & $6.884 \times 10^{17}$ & $5.094 \times 10^{17}$ & \\
& 3 & 1.120 & 1.524 & 0.056 & 1.468 & $1.987 \times 10^{17}$ & $6.718 \times 10^{16}$ & \\
& 4 & 0.713 & 0.930 & 0.044 & 0.887 & $8.544 \times 10^{16}$ & $1.301 \times 10^{16}$ & \\
\hline \hline
\end{tabular}

efficiency. For example, the 2nd subcell in Sharp ${ }^{\circledR}$ device happens to absorb the smallest portion of the solar spectra, Fig. 5 (b), hence has the lowest $J_{\mathrm{sc}}^{(2)}$, Fig. 4 (b). On the other hand the 3rd subcell absorbs the unnecessarily large portion of sun spectra and produces too large $J_{\mathrm{sc}}^{(3)}$ current, detuned from $J_{\mathrm{sc}}^{(2)}$ that defines the overall output current. The excess number of photons only contributes to the losses that reduce $V_{\text {oc }}$ and undermines the efficiency. To correct this, our model reveals that the 3rd subcell should be made of material with larger energy gap, Fig. 5 (a). Such larger gap material will prevent unnecessary absorption of the excess number of photons. This will lead to lower short-circuit current $J_{\mathrm{sc}}^{(3)}$, and in turn will lower losses, Fig. 4 (a). This discussion is supported with results listed in Table 1. The model can be further tested against the set of electrical parameters that can be extracted form measurements on real MJSC devices [35] and compared with our predictions.

The full set of the results of our optimisation procedure, i.e., the optimal $E_{g}$, the optimal thicknesses of subcells, the optimal thicknesses of $p$ - and $n$ regions, $w_{p(n)}$, as well as the optimal acceptor and donor concentrations in each subcell, are summarised in Table 2 . Those data can guide the future experiment and indicate how much more efficiency can be achieved from 2-, 3, and 4- MJSC with targeted $E_{g}$ and other optimised material parameter sets. In Table 2 we can observe the trend that the higher doping concentrations are accompanied with the higher energy gaps, finding correctly the balance between various contradicting requirements imposed by our model for losses. 


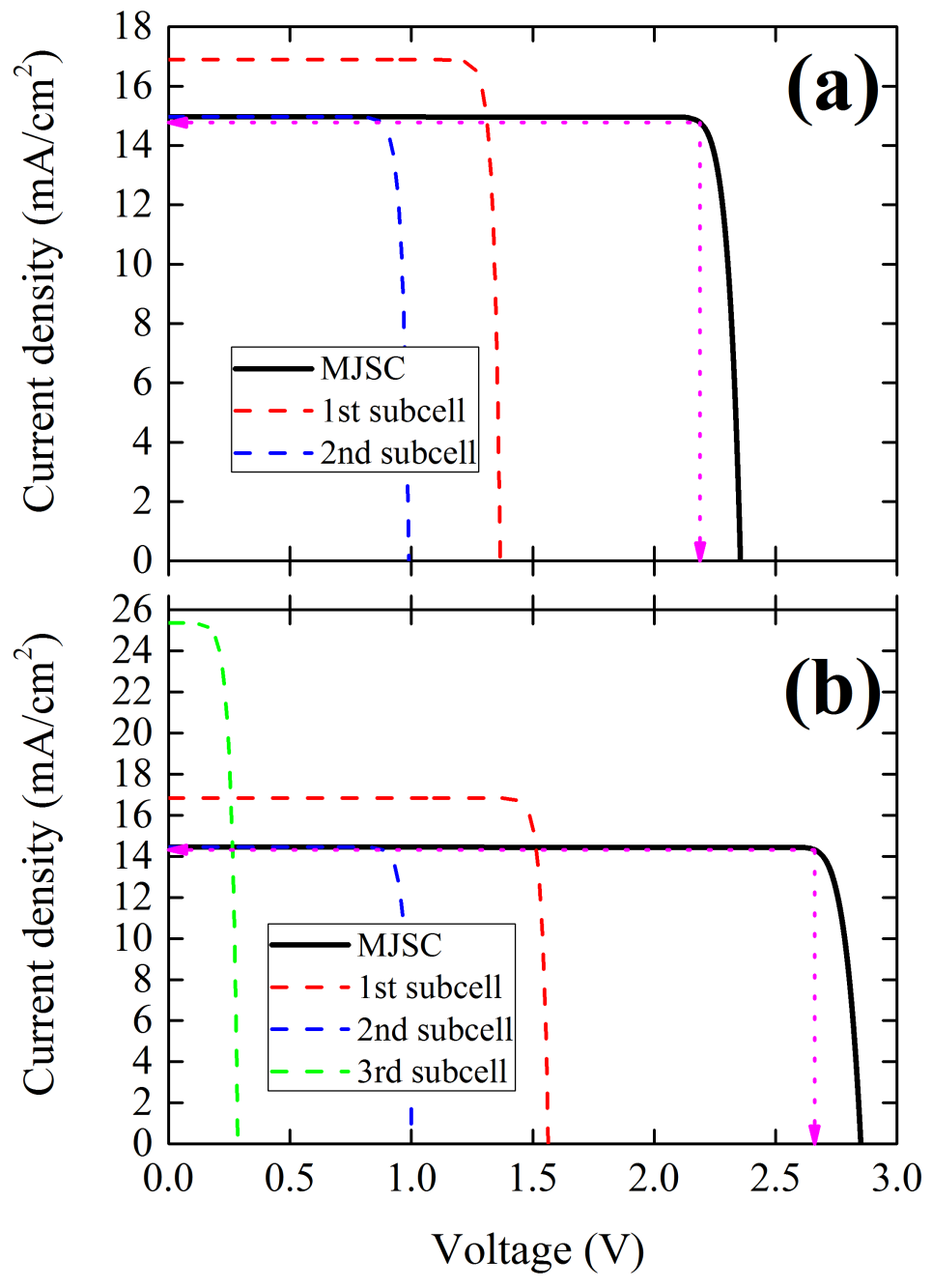

Figure 4: Predicted $J V$ curve of our optimisation based on extracted experimental parameters of actual devices: (a) Alta Devices ${ }^{\circledR}$ 2-junction solar cell, [31] with calculated $J_{\text {opt }}=14.77 \mathrm{~mA} / \mathrm{cm}^{2}$ and $V_{\text {opt }}=2.19 \mathrm{~V}$; (b) Sharp ${ }^{\circledR} 3$-junction solar cell, [32] with calculated $J_{\mathrm{opt}}=14.32 \mathrm{~mA} / \mathrm{cm}^{2}$ and $V_{\mathrm{opt}}=2.66 \mathrm{~V}$. 

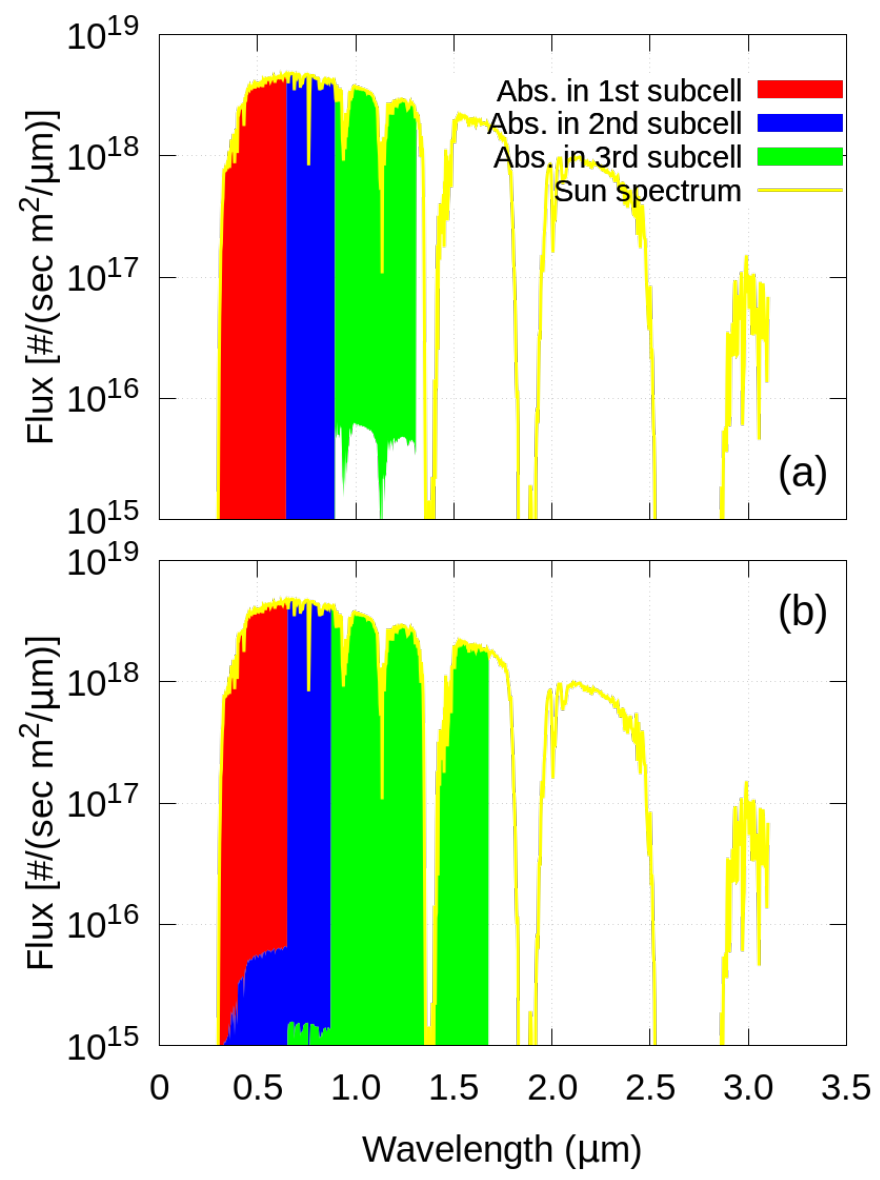

Figure 5: ASTM G173-03 Global tilted solar spectra and attenuated portion of this spectra absorbed by each of 3 subcells in 3-junction SC: (a) optimisation based on our model and (b) optimisation based on real device [32] 
The maximal efficiency of MJSC can be increased by the light concentration. The best MJSC under light concentration are: 2- junction MJSC with $\eta=35.1 \pm 1.1 \%$ under 407 suns [36], 3- junction MJSC with $\eta=44.4 \pm 2.6 \%$ under 302 suns [37], and 4- junction MJSC with $\eta=45.7 \pm 2.3 \%$ under 234 suns [38]. Wafer-bonding and mechanical stacking as methods for SC fabrication proposed by Dimroth et. al. [39, 40], and Essig et. al. [41, 42], allow manufacturing of MJSC with wider range of materials and, thus, more efficient use of solar spectrum. Within this technology, the best 4-junction MJSC is with $\eta=46.0 \pm 2.2 \%$ [43] under 508 suns. Interestingly our algorithm predicts higher efficiency in all cases even for the light concentration of one Sun.

\section{Conclusions}

Although the MJSC exhibits the record SC efficiencies, and is the only concept among the $3^{\text {rd }}$ generation solar cells with proven efficiency that ex-

ceed the SQ limit, they still have not reached their full potential. One of the main reasons is either inadequate parameter selection or limitations in material availability. The consequences are the spectral matching that still deviates from the ideal and sometimes losses are higher than desired. To address these problems, we have presented the heuristic method based on the GA in order to predict the main physical parameters of MJSC devices that will maximise its efficiency. In our model we have considered the radiative as well as non radiative losses, i.e., the diffusion dark current and the Auger losses. The model is applied to III-V semiconductor materials in which all relevant material parameters are scaled or functions of $E_{g}$. The presented model provides a detailed description of processes in MJSC without any fittings and the GA allows for the rapid search through the vast parameter space. As an output, the model predicts the optimal energy gaps and other relevant parameters like impurity concentrations and optimal subcell thicknesses for MJSC under current matching conditions and under 1-Sun. MJSC parameters obtained here can be used as a recipes to experimentalists that could be followed in order to fabricate MJSC devices with improved efficiencies. Since the fabrication method is not specified in our model, only the pnjunctions were optimised, the epitaxial growth, mechanical stacking or wafer bonding can be applied to grow the target structures presented here. 


\section{Appendix}

In III-V semiconductors all basic material parameters like, effective masses, effective density of states and intrinsic carrier concentrations, can be scaled to the energy gap of material. These relations can be derived from the $\mathbf{k} \cdot \mathbf{p}$ theory. The expressions for the electron effective mass is:

$$
m_{e}^{*}\left(E_{g}\right)=m_{0}\left[1+\frac{2 P^{2}}{3 m_{0}}\left(\frac{2}{E_{g}}+\frac{1}{E_{g}+\Delta}\right)\right]^{-1}
$$

where $m_{0}$ is the free electron mass, $E_{g}$ the energy gap $\Delta$ the spin-orbit splitting and $P=-i \frac{\hbar}{m_{0}}\langle s|\hat{\mathbf{p}}| v\rangle$, is the optical dipole matrix element between conduction and valence band states in $\Gamma$ point. In the nearly free electron gas model its magnitude is approximated with $P \approx \hbar\left(2 \pi / a_{0}\right)$, where $a_{0}$ is the lattice constant of the III-V material. Across the family of III-V materials, $2 P^{2} / m_{0}$ is surprisingly constant, [44] and good approximation is to choose its value in the range between 21-26 eV. Next, the expressions for the light hole $m_{\mathrm{lh}}^{*}$, heavy hole $m_{\mathrm{hh}}^{*}$ and spin-orbit interaction $m_{\mathrm{so}}^{*}$ effective masses are:

$$
\begin{gathered}
m_{\mathrm{lh}}^{*}\left(E_{g}\right)=m_{0}\left[1+\frac{2 P^{2}}{m_{0}}\left(\frac{1}{3}\right)\left(\frac{2}{E_{g}}\right)\right]^{-1} \\
m_{\mathrm{hh}}^{*}\left(E_{g}\right) \approx m_{0} / 2 \\
m_{\mathrm{so}}^{*}\left(E_{g}\right)=m_{0}\left[1+\frac{2 P^{2}}{m_{0}}\left(\frac{1}{3}\right)\left(\frac{1}{E_{g}+\Delta}\right)\right]^{-1}
\end{gathered}
$$

Further, effective density of states in conduction $N_{c}$ and valence $N_{v}$ band are given as:

$$
N_{c(v)}\left(E_{g}\right)=2\left(\frac{2 \pi m_{\mathrm{e}(\mathrm{h})}^{*}\left(E_{g}\right) k_{\mathrm{B}} T}{h^{2}}\right)^{3 / 2}
$$

where $m_{\mathrm{h}}^{*}\left(E_{g}\right)=\left[m_{\mathrm{lh}}^{*}\left(E_{g}\right)^{3 / 2}+m_{\mathrm{hh}}^{*}\left(E_{g}\right)^{3 / 2}\right]^{2 / 3}$. The intrinsic carrier concentration $n_{i}$ is then:

$$
n_{i}\left(E_{g}\right)=\sqrt{N_{c}\left(E_{g}\right) N_{v}\left(E_{g}\right)} e^{-E_{g} / 2 k_{\mathrm{B}} T} .
$$

In III-V semiconductors the relation between the refractive index, $\bar{n}$, and the energy gap, $E_{g}$, can be approximated as: 

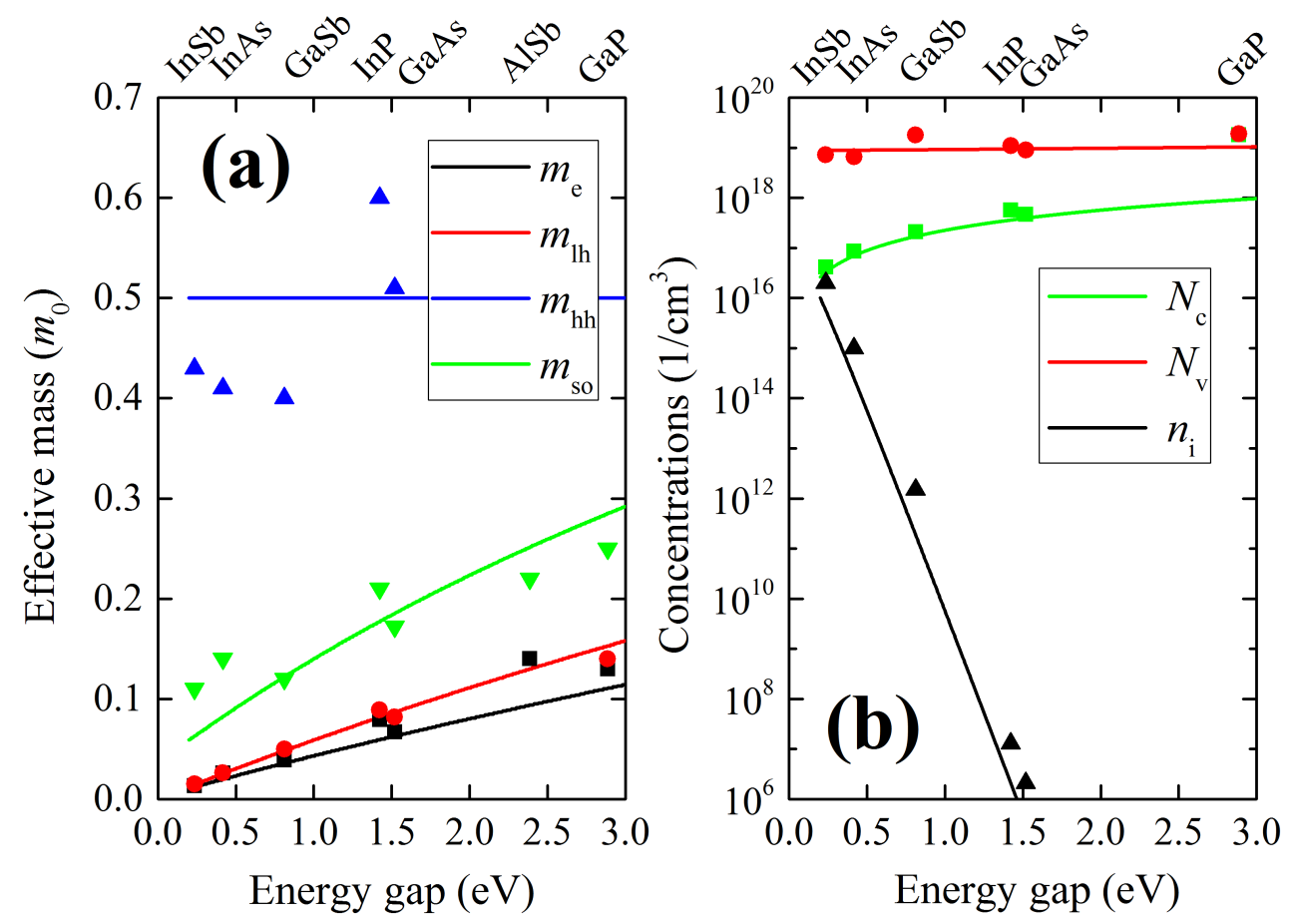

Figure 6: (a) Measured [24, 25] and calculated effective masses for several characteristic III-V materials. (b) Measured [24, 25] and calculated effective densities of states and intrinsic carrier concentration for several III-V semiconductors.

$$
\bar{n}=K E_{g}^{C}
$$

where $K=3.3668$ and $C=-0.32234$. Those constants are obtained from the regression fitting method used on the large set of experimental data on refractive index of semiconducting and oxide materials, and it is valid in the region $E_{g} \in[0.1-8.5] \mathrm{eV}$. [45]. The relative permeability in Eqs. 12 and 14 is then taken as $\varepsilon_{\mathrm{r}}=\bar{n}^{2}$.

\section{Acknowledgements}

The authors wish to thank the NEDO Agency, Japan, for financial support under grant: "Research and Development on Innovative Solar Cells: Post-Silicon solar cells for ultra-high efficiencies," and the contribution of COST Action MP1406 (MultiscaleSolar). ST wish to acknowledge the Royal 
Society, London, grant "High Performance Computing in Modelling of Innovative Photo-Voltaic Devices."

[1] R. R. King, D. C. Law, K. M. Edmondson, C. M. Fetzer, G. S. Kinsey, H. Yoon, R. A. Sherif, and N. H. Karam, "40\% efficient metamorphic GaInP/GaInAs/Ge multijunction solar cells," Appl. Phys. Lett. 90, 18 (2007)

[2] W. Shockley, H.J. Queisser "Detailed balance limit of efficiency of pn junction solar cells" Journal of Applied Physics, 32(3), pp. 510-519, 1961.

[3] R.R. King , "Multijunction cells: Record breakers," Nat. Photon., 2, pp. 284-286, 2008.

[4] A. Brown and M. Green, "Detailed balance limit for the series constrained two terminal tandem solar cell" Physica E, 14, pp. 96-100, 2002 .

[5] G.L. Araujo and A. Marti, "Absolute limiting efficiencies for photovoltaic energy conversion," Solar Energy Materials and Solar Cells 33(2), pp. 213-240, 1994.

[6] A. De Vos , "Detailed balance limit of the efficiency of tandem solar cells" J. Phys. D: Appl. Phys., 13, pp. 839-846, 1980.

[7] P. Würfel, "Thermodynamic limitations to solar energy conversion" Physica E 14, pp. 18-26, 2002.

[8] D. Alonso-Alvarez, T. Wilson, P. Pearce, M. Führer, D. Farrell, N. Ekins-Daukes, "Solcore: A multi-scale, Python-based library for modelling solar cells and semiconductor materials," arXiv:1709.06741 [physics.app-ph]

[9] S. P. Philipps and A. W. Bett, "III-V Multi-junction Solar Cells", Chapter IV in Advanced Concepts in Photovoltaics (Eds. A. J. Nozik, G. Conibeer, and M. C. Beard, The Royal Society of Chemistry, 2014).

[10] L.C. Hirst and N.J. Ekins-Daukes, "Fundamental losses in solar cells" Prog. Photovolt: Res. Appl. 19, pp. 286-293, 2011. 
[11] R. R. King, D. Bhusari, A. Boca, D. Larrabee, X.-Q. Liu, W. Hong, C. M. Fetzer, D. C. Law and N. H. Karam, "Band gap-voltage offset and energy production in next-generation multijunction solar cells," Prog. Photovolt: Res. Appl. 19, 797-812 (2011)

[12] M. C. A. York and S. Fafard, "High efficiency phototransducers based on a novel vertical epitaxial heterostructure architecture (VEHSA) with thin p/n junctions," J. Phys. D: Appl. Phys. 50, 173003 (2017)

[13] S. Fafard, M. C. A. York, F. Proulx, C. E. Valdivia, M. M. Wilkins, R. Ares, V. Aimez, K. Hinzer, and D. P. Masson, "Ultrahigh efficiencies in vertical epitaxial heterostructure architectures," Appl. Phys. Lett. 108, 071101 (2016)

[14] F. Proulx, M. C. A. York, P. O. Provost, R. Ares, V. Aimez, D. P. Masson, S. Fafard, "Measurement of strong photon recycling in ultra-thin GaAs n/p junctions monolithically integrated in high-photovoltage vertical epitaxial heterostructure architectures with conversion efficiencies exceeding 60\%," physica status solidi (RRL) - Rapid Research Letters 11, 1600385 (2017).

[15] Y.P. Varshni, "Band-to-Band Radiative Recombination in Groups IV, VI, and III-V Semiconductors," phys. stat. sol. (b), 20 pp. 9-36, (1967).

[16] J. Blackmore, Semiconductor Statistics (Elsevier Science B.V., 1962).

[17] S. Fonash, Solar Cell Device Physics (Academic Press, 1981).

[18] V. Abakumov, V. Perel, and I. Yassievich, Modern problems in condensed matter sciences, V33 - Nonradiative recombination in semiconductors (1991).

[19] http://rredc.nrel.gov/solar/spectra/am1.5/astmg173/astmg173.html

[20] S. Tomić, A.G. Sanderlend, I.J. Bush, "Parallel multi-band $\mathbf{k} \cdot \mathbf{p}$ code for electronic structure of zinc blend semiconductor quantum dots" $J$. Mater. Chem., 16, pp. 1963-1972, 2006.

[21] J. Nelson, The Physics of solar cells (Imperial College Press, 2003).

[22] H.J. Hovel Semiconductors and Semimetals, V11 - Solar Cells (Academic Press, 1975). 
[23] S.M. Sze, Semiconductor Devices: Physics and Technology (Wiley).

[24] I. Vurgaftman and J. R. Meyer, J. Appl. Phys. 94, 3675 (2003).

[25] http://www.ioffe.ru/SVA/NSM/Semicond/index.html .

[26] A. Mirčetić, D. Indjin, Z. Ikonić, P. Harrison, V. Milanović, R. W. Kelsall, "Towards automated design of quantum cascade lasers," Journal of Applied Physics 97, 084506 (2005).

[27] M. Mitchell,An Introduction to Genetic Algorithms (MIT Press, Cambridge, Massachusetts, 1997).

[28] D. Goldberg,Genetic Algorithms in Search, Optimization, and Machine Learning (Addison-Wesley, Reading, Massachusetts, 1989).

[29] T. Markvart, L. Castañer, Practical Handbook of Photovoltaics: Fundamentals and Applications (Elsevier Science, 2003).

[30] M.A. Green, Y. Hishikawa, W. Warta, et al. "Solar cell efficiency tables (version 50)," Prog Photovolt Res Appl. 25 668-676, 2017.

[31] B.M Kayes, L. Zhang, R. Twist, I.K. Ding, G.S. Higashi, "Flexible thin-film tandem solar cells with $>30 \%$ efficiency" IEEE Journal of Photovoltaics 4 pp. 729-733, 2014.

[32] K. Sasaki, T. Agui, K. Nakaido, N. Takahashi, R. Onitsuka, T. Takamoto, "Development of InGaP/GaAs/InGaAs inverted triple junction concentrator solar cells" AIP Conference Proceedings, 1556 pp. 22-25, 2013.

[33] O.D. Miller ,E. Yablonovitch, S.R. Kurtz, "Strong Internal and External Luminescence as Solar Cells Approach the Shockley-Queisser Limit," IEEE Journal of Photovoltaics 2, pp. 303-311, 2012.

[34] E. Yablonovitch, "Lead halides join the top optoelectronic league," Science, 351, pp. 1401, 2016.

[35] T. Sogabe, A. Ogura, O. Mitsuyoshi, and Y. Okada, "Self-consistent electrical parameter extraction from bias dependent spectral response measurements of III-V multi-junction solar cells," Prog. Photovolt: Res. Appl. 23, 37-48, (2014) 
[36] J. Ohlmann, D. Lackner, J.F. Sanchez, M. Zedda, A. Wekkeli, M. Steiner, A. Fallisch, F. Dimroth, "35.1\% efficient dual junction solar cells optimized for direct hydrogen generation," 44th IEEE PVSC, Washington, 2017.

[37] Press Release, Sharp Corporation, 31 May 2012 (accessed at http://sharpworld.com/corporate/news/120531.html on 5 June 2013).

[38] NREL Press Release NR4514, 16 December 2014.

[39] F. Dimroth, T. N. D. Tibbits, M. Niemeyer, F. Predan, P. Beutel, C. Karcher, E. Oliva, G. Siefer, D. Lackner, P. Fu--Kailuweit, A. W. Bett, R. Krause, C. Drazek, E. Guiot, J. Wasselin, A. Tauzin, and T. Signamarcheix, IEEE Journal of Photovoltaics 6, 343 (2016).

[40] F. Dimroth, T. Roesener, S. Essig, C. Weuffen, A. Wekkeli, E. Oliva, G. Siefer, K. Volz, T. Hannappel, D. Hussler, W. Jger, and A. W. Bett, IEEE Journal of Photovoltaics 4, 620 (2014).

[41] S. Essig, M. A. Steiner, C. Alleb, J. F. Geisz, B. Paviet-Salomon, S. Ward, A. Descoeudres, V. LaSalvia, L. Barraud, N. Badel, A. Faes, J. Levrat, M. Despeisse, C. Ballif, P. Stradins, and D. L. Young, IEEE Journal of Photovoltaics 6, 1012 (2016).

[42] S. Essig, J. Benick, M. Schachtner, A. Wekkeli, M. Hermle, and F. Dimroth, IEEE Journal of Photovoltaics 5, 977 (2015).

[43] Press Release, Fraunhofer Institute for Solar Energy Systems, 1 December 2014 (accessed at http://www.ise.fraunhofer.de/en/ 7 December 2014).

[44] C. Hermann and C. Weisbuch, Phys. Rev. B 15, 823 (1977).

[45] V. Kumar and J. K. Singh, Ind. J. Pure. Appl. Phys. 48, 571 (2010). 